\title{
There is no simple lateral ankle sprain
}

\author{
G. M. M. J. Kerkhoffs ${ }^{1,2,3}$ • J. G. Kennedy ${ }^{4,5}$ J. D. F. Calder ${ }^{6,7} \cdot$ J. Karlsson $^{8}$
}

Published online: 29 March 2016

(C) European Society of Sports Traumatology, Knee Surgery, Arthroscopy (ESSKA) 2016

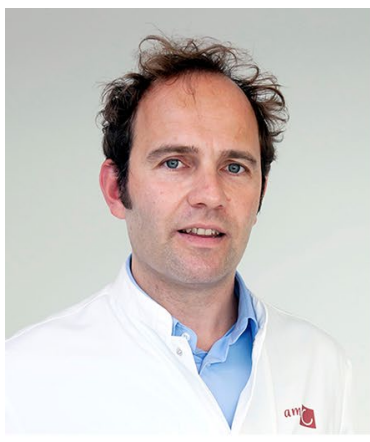

Kerkhoffs G.M.M.J.

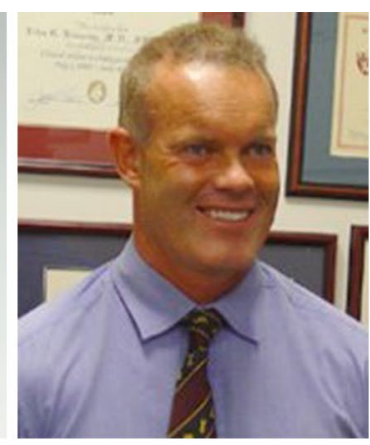

Kennedy J.

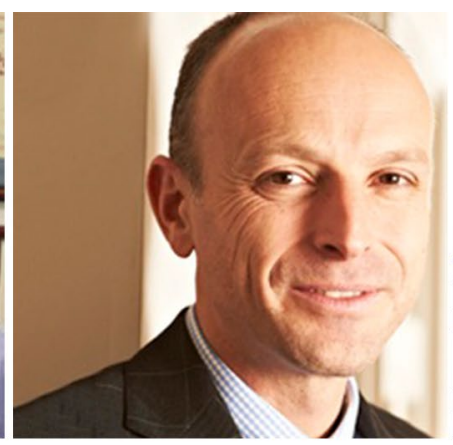

Calder J.

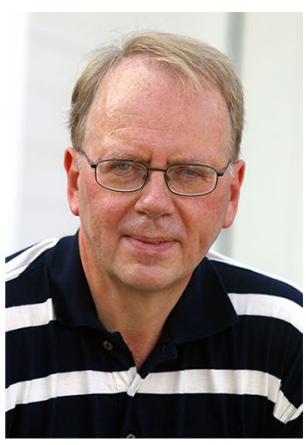

Karlsson J.
KSSTA is proud to present a special issue on ankle joint disorders following a lateral ankle sprain. Lateral ankle

G. M. M. J. Kerkhoffs

g.m.kerkhoffs@amc.uva.nl

1 Department of Orthopedic Surgery, Academic Medical Center, University of Amsterdam, Meibergdreef 9, 1105 AZ Amsterdam, The Netherlands

2 Academic Center for Evidence Based Sports Medicine (ACES), Meibergdreef 9, 1105 AZ Amsterdam, The Netherlands

3 Amsterdam Collaboration for Health and Safety in Sports (ACHSS), AMC/VUmc IOC Research Center, Meibergdreef 9, 1105 AZ Amsterdam, The Netherlands

4 Hospital for Special Surgery, New York, NY, USA

5 Weil Cornell University, New York, NY, USA

6 Fortius Clinic, London, UK

7 Imperial College, London, UK

8 Department of Orthopaedics, Sahlgrenska University Hospital, Sahlgrenska Academy, Gothenburg University, Gothenburg, Sweden sprains remain the single most frequent injury in modern sports. With the growing knowledge on ankle joint disorders, it appears that this injury causes more damage to the ankle joint than was previously assumed. Previously, few papers have investigated the concomitant injuries that may be associated with a "simple lateral ankle sprain". The editors have selected peer-reviewed articles for this current special edition, which present updated scientific information on the pathologies associated with an ankle sprain. We hope that the reader will be in a position, after reading this edition, to incorporate this information into common daily practice when managing ankle injuries and in this way reduce the morbidity associated with this seemingly innocuous condition. The cornerstone of clinical practice is sound knowledge of surgical anatomy. For this reason, we have decided to republish Golano's article on the anatomy of the ankle ligaments as the leading paper in this issue with an addendum from one of Golano's former co-workers, Mik Dalmau Pastor, representing the Barcelona School of Orthopaedic Surgical Anatomy [5]. 
In addition to the traditional diagnosis and treatment strategies for ankle sprains commonly described, this volume also explores new studies optimising the early management of these injuries. Bekerom et al. [14] present the results of a randomised controlled trial on functional treatment for lateral ankle ligament injuries and they also report on a systematic review on the value of the use of non-steroidal anti-inflammatory drugs (NSAIDs) [15]

Several studies report on the results of open surgical treatment for ligament injuries in both the chronic and acute setting; White et al. [23] report that a high number of their professional athletes return to sports after early open repair of acute injuries. Potential beneficial results of an arthroscopic lateral ligament reconstruction over the classical open reconstruction have been extensively discussed at congresses in recent years. As a consequence of the emerging controversies in arthroscopic versus open repair, there are several manuscripts detailing the relative merits of one technique over the other. This includes novel arthroscopic visualisation of the lateral ankle ligaments [13], a biomechanical analysis of an all-inside arthroscopic modified Broström operation for chronic lateral ankle instability [9] and the first results of an arthroscopic anatomical reconstruction by Guillo et al. [6].

A lateral ankle sprain may also cause pathology in the peroneal tendons, as well as a rupture of the superior peroneal retinaculum resulting in a peroneal tendon dislocation. Two systematic reviews provide an overview of the results of patients treated for peroneal tendon dislocation as well as proposing a rehabilitation programme after surgical treatment for peroneal tendon ruptures $[18,19]$.

The vascularity of the peroneal tendon, often implicated in its poor reparative potential, has been elegantly described in an anatomical study leading the section on peroneal tendon disorders [20].

The research group from Vail, Colorado, USA, presents an interesting paper on the radiographic identification of the primary structures of the ankle syndesmosis. This paper is the leading paper of the section on syndesmotic injuries [22]. The Ankle and Foot Associates (ESSKAAFAS) also present consensus papers on the classification and management of acute isolated syndesmotic injuries $[16,17]$.

A lateral ankle sprain may cause a cartilage injury as the medial talar dome impacts on the distal tibial plafond. This may initiate the development of an osteochondral cyst through cracks in the subchondral plate, destabilise a previously stable osteochondritis dissecans lesion or create an osteochondral fracture leading to chronic pain from a talar osteochondral lesion (OCL). Different aspects of the morphological findings in juvenile OCLs are presented by Roßbach et al. [10] comparing MRI findings with arthroscopic findings. Results of various surgical treatment protocols for primary OCLs are presented-ranging from autologous osteochondral transplantation [4] to the Lift Drill Fill Fix (LDFF) procedure, a new arthroscopic talar cartilage preserving technique [8].

The vast majority of arthritic disease in the ankle joint is post-traumatic, following either a sprain or a fracture. Therefore, this current edition also examines treatment strategies for end-stage post-traumatic arthritis of the ankle. Franceschi et al. [3] present a systematic review of the results of ankle fusion with an intramedullary nail, and the Amsterdam school presents medium-term results of posterior arthroscopic ankle fusion for post-traumatic ankle osteoarthritis [2, 7].

With the vast increase in the number of ankle surgeries, there is need for evidence-based knowledge on the prevention of venous thromboembolism (VTE) in ankle surgery and this is provided by a meta-analysis by Calder et al. [1].

In order to adequately measure the outcome of orthopaedic interventions, it is increasingly important to validate the outcome scores being used, and therefore, the last section is devoted to a discussion about validation and responsiveness of scoring systems. Important changes are also made to the Dutch version of the Foot and Ankle Outcome Score (FAOS) $[11,12,21]$.

This special issue is composed of research manuscripts that have attempted to describe, evaluate and further the treatment of the "simple lateral ankle sprain". Collectively we hope that they will help the reader continue to pursue clinical excellence in treating these injuries. In addition, we hope that this volume will stimulate further debate, research and ultimately improve the management for this common sport-related injury in the future.

\section{References}

1. Calder JDF, Freeman R, Domeij, Arverud E, van Dijk CN, Ackermann PW. Meta-analysis and suggested guidelines for prevention of venous thromboembolism (VTE) in foot and ankle surgery. Knee Surg Sports Traumatol Arthrosc. doi:10.1007/ s00167-015-3976-y

2. de Leeuw PAJ, Hendrickx RPM, van Dijk CN, Stufkens SS, Kerkhoffs GMMJ (2016) Midterm results of posterior arthroscopic ankle fusion. Knee Surg Sports Traumatol Arthrosc. doi:10.1007/s00167-015-3975-Z

3. Franceschi F, Franceschetti E, Torre G, Papalia R, Samuelsson K, Karlsson J, Denaro V (2016) Tibiotalocalcaneal arthrodesis using an intramedullary nail: a systematic review. Knee Surg Sports Traumatol Arthrosc. doi:10.1007/s00167-015-3548-1

4. Fraser EJ, Harris MC, Prado MP, Kennedy JG (2015) Autologous osteochondral transplantation for osteochondral lesions of the talus in an athletic population. Knee Surg Sports Traumatol Arthrosc. doi:10.1007/s00167-015-3606-8

5. Golanó P, Vega J, de Leeuw PA, Malagelada F, Manzanares MC, Götzens V, van Dijk CN (2010) Anatomy of the ankle ligaments: a pictorial essay. Knee Surg Sports Traumatol Arthrosc 18(5):557-569 
6. Guillo S, Takao M, Calder J, Karlson J, Michels F, Bauer $\mathrm{T}$ (2015) Arthroscopic anatomical reconstruction of the lateral ankle ligaments. Knee Surg Sports Traumatol Arthrosc. doi:10.1007/s00167-015-3789-z

7. Hendrickx RPM, de Leeuw PAJ, Golano P, van Dijk CN, Kerkhoffs GMMJ (2014) Safety and efficiency of posterior arthroscopic ankle arthrodesis. Knee Surg Sports Traumatol Arthrosc. doi:10.1007/s00167-014-3040-3

8. Kerkhoffs GMMJ, Reilingh ML, Gerards RM, de Leeuw PAJ (2014) Lift, drill, fill and fix (LDFF): a new arthroscopic treatment for talar osteochondral defects. Knee Surg Sports Traumatol Arthrosc. doi:10.1007/s00167-014-3057-7

9. Lee KT, Kim ES, Kim YH, Ryu JS, Rhyu IJ, Lee YK (2014) All-inside arthroscopic modified Broström operation for chronic ankle instability: a biomechanical study. Knee Surg Sports Traumatol Arthrosc. doi:10.1007/s00167-014-3159-2

10. Roßbach BP, Paulus AC, Niethammer TR, Wegener V, Gülecyüz MF, Jansson V, Müller PE, Utzschneider S (2015) Discrepancy between morphological findings in juvenile osteochondritis dissecans (OCD): a comparison of magnetic resonance imaging (MRI) and arthroscopy. Knee Surg Sports Traumatol Arthrosc. doi:10.1007/s00167-015-3724-3

11. Sierevelt IN, Beimers L, van Bergen CJA, Haverkamp D, Terwee CB, Kerkhoffs GMMJ (2014) Validation of the Dutch language version of the Foot and Ankle Outcome Score. Knee Surg Sports Traumatol Arthrosc. doi:10.1007/s00167-014-3017-2

12. Sierevelt IN, van Eekeren ICM, Haverkamp D, Reilingh ML, Terwee CB, Kerkhoffs GMMJ (2015) Evaluation of the Dutch version of the Foot and Ankle Outcome Score (FAOS): responsiveness and minimally important change. Knee Surg Sports Traumatol Arthrosc. doi:10.1007/s00167-015-3941-9

13. Thès A, Klouche S, Ferrand M, Hardy P, Bauer T (2015) Assessment of the feasibility of arthroscopic visualization of the lateral ligament of the ankle: a cadaveric study. Knee Surg Sports Traumatol Arthrosc. doi:10.1007/s00167-015-3804-4

14. van den Bekerom MPJ, van Kimmenade R, Sierevelt IN, Eggink K, Kerkhoffs GMMJ, van Dijk CN, Raven EEJ (2015) Randomized comparison of tape versus semi-rigid and versus lace-up ankle support in the treatment of acute lateral ankle ligament injury. Knee Surg Sports Traumatol Arthrosc. doi:10.1007/ s00167-015-3664-y
15. van den Bekerom MPJ, Sjer A, Somford MP, Bulstra GH, Struijs PAA, Kerkhoffs GMMJ (2014) Non-steroidal anti-inflammatory drugs (NSAIDs) for treating acute ankle sprains in adults: benefits outweigh adverse events. Knee Surg Sports Traumatol Arthrosc. doi:10.1007/s00167-014-2851-6

16. van Dijk CN, Longo UG, Loppini M, Florio P, Maltese L, Ciuffreda M, Denaro V (2016) Conservative and surgical management of acute isolated syndesmotic injuries: ESSKA-AFAS consensus and guidelines. Knee Surg Sports Traumatol Arthrosc. doi:10.1007/s00167-016-4017-1

17. van Dijk CN, Longo UG, Loppini M, Florio P, Maltese L, Ciuffreda M, Denaro V (2015) Classification and diagnosis of acute isolated syndesmotic injuries: ESSKA-AFAS consensus and guidelines. Knee Surg Sports Traumatol Arthrosc. doi:10.1007/ s00167-015-3942-8

18. van Dijk PAD, Lubberts B, Verheul C, DiGiovanni CW, Kerkhoffs GMMJ (2016) Rehabilitation after surgical treatment of peroneal tendon tears and ruptures. Knee Surg Sports Traumatol Arthrosc. doi:10.1007/s00167-015-3944-6

19. van Dijk PAD, Gianakos AL, Kerkhoffs GMMJ, Kennedy JG (2015) Return to sports and clinical outcomes in patients treated for peroneal tendon dislocation: a systematic review. Knee Surg Sports Traumatol Arthrosc. doi:10.1007/s00167-015-3833-z

20. van Dijk PAD, Madirolas FX, Carrera A, Kerkhoffs GMMJ, Reina F (2016) Peroneal tendons well vascularized: results from a cadaveric study. Knee Surg Sports Traumatol Arthrosc. doi:10.1007/s00167-015-3946-4

21. Weel H, Zwiers R, Azim D, Sierevelt IN, Haverkamp D, van Dijk CN, Kerkhoffs GMMJ (2014) Validity and reliability of a Dutch version of the foot and ankle ability measure. Knee Surg Sports Traumatol Arthrosc. doi:10.1007/s00167-014-3480-9

22. Williams BT, James EW, Jisa KA, Haytmanek CT, LaPrade RF, Clanton TO (2015) Radiographic identification of the primary structures of the ankle syndesmosis. Knee Surg Sports Traumatol Arthrosc. doi:10.1007/s00167-015-3743-0

23. White JW, McCollum GA, Calder JDF (2015) Return to sport following acute lateral ligament repair of the ankle in professional athletes. Knee Surg Sports Traumatol Arthrosc. doi:10.1007/s00167-015-3815-1 